\title{
An Effective Interpretation of Wave Function of Microscopic Particles
}

Change Zhang ( $\nabla$ change.zhang10@gmail.com )

Institute of Theoretical Physics

\section{Research Article}

Keywords: Quantum mechanics, Wave-particle duality, Quantum entanglement, Cosmic redshift

Posted Date: December 1st, 2020

DOI: https://doi.org/10.21203/rs.3.rs-113149/v1

License: (c) (i) This work is licensed under a Creative Commons Attribution 4.0 International License. Read Full License 


\title{
An Effective Interpretation of Wave Function of Microscopic
}

\section{Particles}

\author{
Change Zhang \\ Institute of Theoretical Physics, Chinese Academy of Sciences, Beijing 100190, P. R. China \\ E-mail: change.zhang10@gmail.com
}

\begin{abstract}
Because the origin of the wave-particle duality of quantum mechanics has always been an unsolved problem in physics. Therefore, this article attempts to propose a feasible interpretation of the wave-particle duality origin of quantum mechanics in order to eliminate the confusion of probability waves in quantum mechanics.
\end{abstract}

\section{Keywords}

Quantum mechanics; Wave-particle duality; Quantum entanglement; Cosmic redshift;

\section{1: Introduction}

The controversy over the volatility and particle nature of light has been a problem that has continued since the Newtonian era. It was not until the establishment of quantum mechanics in the last century that we realized that light and other microscopic particles have wave-particle duality. However, what is the origin of wave-particle duality? How to interpret the confusion of quantum mechanical probability waves? What is the origin of quantum and quantum entanglement? Through the existing quantum theory, we still can't get a good explanation, so this paper tries to perfect the existing quantum mechanics theory to solve these problems. At the same time, looking at the relevant literature, we can understand that predecessors have made a lot of efforts in these fields [1-16]. Therefore, I also want to contribute in my own way.

In the latest research, I found that there are time intervals in the vibration of all different kinds of waves, and the time interval will cause the radiated energy to be discrete (discontinuous). In other words, the discreteness of energy can be used as an inherent property of all different kinds of waves. In this way, we can explain the particle nature of light from the perspective of light volatility, and thus propose a feasible way of explaining the origin of the wave-particle duality of light.

In addition, for the motion of objects, we found that discussing the position and momentum of objects is only applicable to macroscopic objects. For the motion of microscopic matter particles, we are only suitable to discuss its energy flow density (or scattering density), not its position and momentum. If we forcefully discuss the position and momentum of microscopic matter particles, it will inevitably lead to probability waves. And if we change our perspective to discuss the energy flux density or scattering density of microscopic matter particles, we can explain the origin of the wave-particle duality of microscopic matter particles and eliminate the confusion of probability waves of microscopic matter particles.

So, this paper attempts to propose a feasible way to explain the origin of quantum mechanics wave-particle duality, in order to eliminate the confusion of quantum mechanics probability waves, and got some new predictions.

\section{2: A new interpretation of the wave-particle duality of light}

According to quantum optics [17], electrons make irregular periodic motion around the core (electron cloud model), while photons (light waves) are generated by electron radiation. Because the mass of the electron after radiating the photon does not cause loss, so if there is no medium, the electron will not be able to transfer energy to the outside. Then, we can put forward a conjecture that light may be a medium wave. Therefore, the frequency $v$ of the light wave is equal to the number $n$ of electrons orbiting the nucleus per unit time.

$$
v=n=\frac{1}{T}
$$

The kinetic energy of an electron that is transmitted to the medium through collisions can be defined as a constant, which we call Planck constant $h$ here. Due to the irregular periodic movement of electrons around the core, there is a time interval between the running cycles, and then the energy they radiate is 
not continuous, but can only be a separate energy, so the energy of the light wave is:

$$
E=h n=h v
$$

It can be seen from the above that the origin of the light quantum is the energy $E=h n=h v \quad(n=v=1)$ of a light wave with a frequency of $n=v=1$ generated by one cycle of the electron. In other words, the origin of quantum is the kinetic energy of a single wave. In other words, "quantum" can also be called "wave element".

The photoelectric effect of the current generated by the energy of the photon absorbed by the electron is:

$$
h v=E_{k}+w
$$

It can be seen from the above that the irregular periodic movement of electrons around the core will inevitably cause the energy of its radiation and absorption to be discrete rather than continuous. Thus, we can explain the particle nature of light from the perspective of the wave nature of light. Because we can explain the particle nature of light from the perspective of the wave nature of light, we explain the origin of the wave-particle duality of light.

Discussion: In fact, not only the energy of light waves is discrete, but the energy of all waves should be discrete.

Because all waves are generated by periodic vibration, there is a time interval for any periodic vibration, and the time interval between vibration and vibration will cause the radiated energy to be discrete. Therefore, theoretically we can measure whether the energy of the sound wave is discrete to obtain experimental verification). In other words, the discontinuity (discreteness) of energy can be an inherent property of waves, not unique to light waves.

Considering that the energy of electron radiation has macro fluctuations, then the Huygens-Fresnel principle is satisfied:

$$
d E=\frac{K d s}{L} \cos \left(\omega t-\frac{2 \pi L}{\lambda}\right)
$$

Combined vibration:

$$
E=\int d E=\int_{S} \frac{K d s}{L} \cos \left(\omega t-\frac{2 \pi L}{\lambda}\right)
$$

Considering that light waves can propagate in vacuum, liquid, gas, and solid, this shows that light is a longitudinal wave of the medium rather than a transverse wave (explained later), and the microscopic mathematical expression can be expressed as:

$$
\tau_{j i, j}+\rho f_{i}=\rho \frac{\partial^{2} u_{i}}{\partial t^{2}}
$$

The wave velocity of the longitudinal wave is:

$$
V=\sqrt{\frac{E}{\rho}}
$$

Since we consider light waves here as longitudinal waves rather than transverse waves, we can only use mathematical expressions of longitudinal waves.

\section{The explanation is as follows:}

Shear wave characteristics for light polarization. We can explain it as follows: When the longitudinal wave of light passes through a solid medium (polarizer), it causes secondary vibration (the solid polarizer becomes a new wave source). Because the polarizer does not shift as a solid medium, only shear deformation occurs. Therefore, light waves are converted from $\mathrm{P}$-waves to $\mathrm{S}$-waves. Therefore, the light wave has the property of a transverse wave. Therefore, we can use the longitudinal wave to explain the polarization phenomenon found by Marius.

Discussion: Traditional theory has always believed that light waves are shear waves, which is actually a misunderstanding. Shear waves require a solid medium, and the existence of solid media will cause stars to fail to move. Astronomical observations show that all stars are moving, so light waves cannot be shear waves. Because light waves are the energy of electronic vibration radiation, the vibration of electrons belongs to mechanical motion, so light waves belong to mechanical waves. (Note: Light is a mechanical wave, a recognition based on 20th century quantum optics [17]. And Marius's law is a theory based on the cognition of the 19th century. Because there was no micro-atomic physics at that time, Marius did not know that light waves were generated by electronic vibrations. Due to the limitations of the cognition of the times, Marius did not know that light waves are mechanical waves).

At the same time, it can be seen from the above formula that the speed of light $V$ is inversely proportional to the density of the medium, expressed as: 


$$
V \propto \frac{1}{\rho}
$$

According to Maxwell's electromagnetic theory, the wave velocity $V$ of the electromagnetic wave is inversely proportional to the relative permittivity $\varepsilon_{r}$

and relative permeability $u_{r}$ of the medium:

$$
V \propto \frac{1}{\mathcal{E}_{r} \boldsymbol{u}_{r}}
$$

Considering that the light wave is a kind of electromagnetic wave, and the light wave is a medium wave, then the electromagnetic wave is also a medium wave, then the relative permittivity $\varepsilon_{r}$ of the medium is proportional to the relative permeability $u_{r}$ and the medium density $\rho$.

$$
\varepsilon_{r} u_{r} \propto \rho
$$

Then the wave velocity of the electromagnetic wave is inversely proportional to the density of the medium:

$$
V \propto \frac{1}{\boldsymbol{E}_{r} \boldsymbol{U}_{r}} \propto \frac{1}{\rho}
$$

Considering that light is a longitudinal wave of the medium. The distribution of the gravitational field of the medium relative to the star in non-flat spacetime. Then, the distribution of the density of the optical medium must be affected by gravity. Thus, a light wave is a wave in which a sparse medium and a dense medium are combined with each other. Then, the medium density is proportional to the gravitational field strength distribution $\rho \propto E$, then the medium density is proportional to the negative value of the gravitational potential gradient:

$$
\rho \propto E=-\nabla \gamma P
$$

Among them, $\gamma$ is an undetermined constant. It can be seen from the above formula that the medium density of light waves is proportional to the field strength. Since the speed of light is inversely proportional to the density of the medium, there is an inverse relationship between the speed of light $V$ and the strength of the gravitational field $E$ as:

$$
V \times E=k(k \neq 0)
$$

Where $k$ is a constant. It can be seen from the above that the stronger the gravitational field strength $E$, the slower the light propagation speed $V$, and at the same time the speed of light presents a gradient distribution in the gravitational field. Therefore, we can prove it experimentally by observing the speed of light at different field strength positions of the same gravitational field and the value of the speed of light propagation near different gravitational field strengths.

Because the density of the optical medium in the strong gravitational field is relatively large, then when a beam of light passes through the strong gravitational field, it belongs to the optical sparse medium and propagates to the optical dense medium. Therefore, when a beam of light passes near a celestial body with a strong gravitational field, the light will inevitably have a significant refraction phenomenon. Of course, this deflection phenomenon has been confirmed by astronomical observation, and its deflection rate satisfies the law of refractive index:

$$
n=\frac{c}{v}
$$

Because the speed of light in the same gravitational field is not a fixed velocity value, but a gradient change value, then the refractive index of light in the same gravitational field should be a range value that varies with the gravitational field strength gradient, which satisfies the relationship:

$$
n \propto \rho \propto E=-\nabla \gamma P
$$

This is consistent with the fact that the deflection of light obtained from actual astronomical observations is a range value. Therefore, we will be surprised to find that this article explains the specific mechanism of light bending predicted by Einstein's general theory of relativity from a microscopic perspective. That is to say, I established a microscopic expression model theory about the bending of light predicted by Einstein's general theory of relativity.

Taking into account the wavelength and refractive 
index also satisfy the following relationship:

$$
\lambda^{\prime}=\frac{\lambda}{n}
$$

Among them, $\lambda$ represents the wavelength in a vacuum, $\lambda^{\prime}$ represents the wavelength in a non-vacuum medium, and $n$ is the refractive index. Thus, we can see that when a beam of light passes near a strong gravitational field, its wavelength becomes smaller because its refractive index becomes larger. And when a beam of light is emitted from the inside of the gravitational field, it belongs to the process of the optical dense medium propagating to the optical sparse medium, the refractive index becomes smaller, then its wavelength will become larger, and a red shift will occur. This can explain the phenomenon of gravitational redshift.

Therefore, we will be surprised to find that this article explains the specific mechanism of gravitational redshift predicted by Einstein's general theory of relativity from a micro perspective. That is to say, I established a micro-expression model theory about the gravitational redshift predicted by Einstein's general theory of relativity.

\section{Discussion and prediction:}

A: Due to the gravitational field distribution of the light's propagating medium relative to the star, then the medium must follow the periodic motion of the gravitational field synchronously. We can observe whether there is a medium perturbation wave with periodic motion through astronomy to prove whether light has the medium, and then verify the rationality of the theory in this paper.

B: If light exists in the medium and is statically distributed relative to the gravitational field. We know that when an object on the earth explodes, a high-density medium shock wave will be generated. The density of the optical medium in the shock wave will inevitably be greater. We can let a beam Light passes through the shock wave and observes whether the speed of light is slow to prove whether there is a medium of light and whether the light is a longitudinal wave combining sparse and dense media.

\section{3: A new interpretation of the wave-particle duality of other matter particles}

As can be seen from the above, light is a medium particle wave, and the light intensity represents the energy flow density passing a certain point. The mathematical expression is:

$$
I=\bar{S}=\bar{\Omega} u=2 \pi^{2} \rho A^{2} v^{2} u
$$

Therefore, there is no physical meaning in discussing the position and momentum of light waves from a micro perspective. Only the distribution of energy flow density at each point in space can be discussed, which is in accordance with the principle of classical wave optics, so there is no probability distribution problem. So, we can eliminate the confusion of the quantum probability distribution of light.

There is a wave diffraction phenomenon when the electron beam passes through some crystals. Since electrons move periodically, their wavelength does not indicate that the electron is a wave. It indicates the orbital distance between the electrons that moved in the previous week and the electrons that moved in the following week (or the distance between the two batches of electrons in translation) during the electronic cycle operation, which satisfies the relationship:

$$
\lambda=\frac{2 \pi r}{n}
$$

Among them, the frequency $v$ represents the number $n$ of electrons moving around the core in unit time (or the batch of electrons passing through a point in space in unit time).

$$
v=n=\frac{2 \pi r}{\lambda}
$$

Therefore, we believe that electrons or other microscopic material particles (such as neutrons, atoms, etc.) will undergo wave diffraction phenomenon through certain crystals. It does not mean that the microscopic material particles are a kind of wave, but that the elastic wave scattering occurs when they pass through the crystal, and this scattering effect reflects a kind of volatility in the macro.

For the mathematical description of the motion state of the scattering particles after the elastic wave scattering of microscopic matter particles, we can use the Schrödinger equation to express:

$$
i \hbar \frac{\partial \psi}{\partial t}=\hat{H} \psi
$$

It reflects the change law of the movement state of the number of scattered particles of microscopic material particles with time after wave scattering. 
For the material wave equation that does not change with time, there is a stationary Schrodinger equation, which is expressed as:

$$
\hat{H} \psi=E \psi
$$

In the same way, the intensity of the material wave (the square of the amplitude) does not represent the probability density that appears somewhere, but the number of scattered particles $N$.

$$
N \propto|\psi(r, t)|^{2}
$$

Thus, we have solved the confusion of probability waves in quantum mechanics. Because the fluctuation of microscopic matter particles is the macroscopic scattering effect of a group of particles, I explain the origin of the wave-particle duality of microscopic matter particles.

\section{4: A new interpretation of quantum entanglement}

Another fascinating problem in quantum mechanics is the origin of quantum entanglement, an unsolved mystery in physics that has not yet been solved.

According to the above, the essence of quantum is the energy of a single wave. Because the potential of a light wave is spherically symmetric $V(r) \psi$, the potential $d v$ at any point on the light wave always has a symmetrical potential point $d v^{\prime}$ in the opposite direction through the center of the sphere. We can think of any such pair of symmetrical potential points as a pair of entangled quanta. Therefore, quantum entanglement is a symmetrical state of two potential points $d v$ and $d v^{\prime}$ on a light wave $\psi$. The mathematical expression is:

$$
d v i d v^{\prime}
$$

When we measure the potential point $d v$ on the light wave, it will cause the spherical symmetry potential $V(r) \psi$ of the light wave to break, which will cause the symmetry of the spherical wave symmetry potential of the light wave to disappear (this is the measurement causing the wave function $\psi$ to collapse), The potential point $d v^{\prime}$ is caused to deviate from their symmetrical state, causing the potential point $d v^{\prime}$ to move. This is the quantum entanglement effect, and we explain the origin of quantum entanglement.

When we measure the potential point on the light $d v$, will result in the light of spherically symmetric potential $V(r) \psi$ damage, leading to the ball of light waves in the symmetrical state of symmetrical potential disappear. (this is measuring led to the collapse of the wave $\psi$ function), causing potential points $d v^{\prime}$ off their symmetrical state, causing potential point $d v^{\prime}$ movement, this is the effect of quantum entanglement, which we explained the origin of the quantum entanglement. From the above analysis, you can see that quantum entanglement is a manifestation of the symmetrical state of the potential of two symmetrical potential points on a wave, which can be used to explain the quantum entanglement of all objects. (If you ask me how I think about this point I put forward? I personally think that this may really be the truth.)

\section{5: Another possible interpretation for the cosmic red-shift}

Since there is a medium for the propagation of light, then the medium of light has kinetic and potential energy, that is, the Hamiltonian $H$. During the propagation of light waves through the medium, the Hamiltonian of the medium needs to be overcome. We define this quantity as the Hamiltonian damping coefficient $\beta$.Therefore, due to the energy loss in the light propagation process, the frequency of the light wave will decrease, satisfying the following mathematical relationship:

$$
T=\frac{2 \pi}{\omega}=\frac{2 \pi}{\left(\omega_{0}^{2}-\beta^{2}\right)^{\frac{1}{2}}}
$$

From the above formula, it can be seen that when energy loss occurs during the propagation of light, the frequency $T$ of the light will decrease, and the spectrum will redly shift. Therefore, the redshift amount $\lambda$ is proportional to the damping coefficient $\beta$ of the Hamiltonian: $\lambda \propto \beta$, and because the damping coefficient $\beta$ of the Hamiltonian is proportional to the distance $\beta \propto r$ of the light, the redshift is proportional to the distance:

$$
\lambda \propto \beta \propto r
$$

Thus, we can use it to explain the cosmic redshift. If the amount of redshift due to frequency reduction within a unit distance is expressed by the constant $k$, then the total redshift $d_{\lambda}$ and the propagation 
distance $r$ satisfy the following relationship:

$$
d_{\lambda}=k \times r
$$

It can be seen from the above that the farther away the light from a star, the greater the energy loss and the greater the redshift. This could provide another feasible explanation for the cosmic spectrum redshift. (If you ask me if I believe my opinion? My answer is, yes, I believe)

Discussion: According to the above analysis, the nature of light as a medium wave will inevitably cause energy loss during its propagation. Because of the work done, the energy lost is converted into heat. Therefore, we can predict that there is a thermal energy temperature in our universe, and this can be used to explain the source of the $3 k$ temperature value that exists in the cosmic microwave background radiation.

\section{6: Another possible interpretation for the invariant principle of the speed of light}

A misunderstood principle of the invariance of the speed of light: according to Galileo's principle of relativity, the mechanical principles in any inertial frame are equivalent. Therefore, for the speed of light, due to the static distribution of the light medium relative to the gravitational field, the speed of light we measure in any gravitational field is a universal velocity value measured with the medium that is stationary relative to the gravitational field as the inertial reference frame. We can define this phenomenon as: the principle of relative velocity invariance. Since light waves propagate from the sun to the earth, they are switched from one gravitational system (constraint system) to another, and there is no separation distance $x^{\prime}=x$ between the two gravitational systems. Therefore, the switching of light waves between two gravitational systems satisfies the relationship:

$$
\left\{\begin{array} { l } 
{ x ^ { \prime } = x } \\
{ y ^ { \prime } = y } \\
{ z ^ { \prime } = z } \\
{ t ^ { \prime } = t }
\end{array} \text { or } \quad \left\{\begin{array}{l}
x=x^{\prime} \\
y=y^{\prime} \\
z=z^{\prime} \\
t=t^{\prime}
\end{array}\right.\right.
$$

The relative velocity invariance principle is obtained by calculating the first derivative of the first three formulas in the above formula with respect to time:

$$
\left\{\begin{array}{l}
v_{x}^{\prime}=v_{x} \\
v_{y}^{\prime}=v_{y} \\
v_{z}^{\prime}=v_{z}
\end{array}\right.
$$

From the above expression, we can predict that the switching of the speed of sound in the two inertial reference frames also satisfies the principle of speed invariance. That is, we can do an experiment of sound velocity propagation between an airplane and the ground. The sound velocity of the sound waves emitted by the inside of the aircraft measured by people on the ground is $340 \mathrm{~m} / \mathrm{s}$, not $580 \mathrm{~m} / \mathrm{s}$ after the superposition of the aircraft's speed of $240 \mathrm{~m} / \mathrm{s}$ and the sound velocity of $340 \mathrm{~m} / \mathrm{s}$.

The reason is that during the propagation of sound waves from the interior of the aircraft to the ground, there is a switching of the media inertial system. When sound waves propagate to the aircraft shell, it will cause secondary vibration of the aircraft shell. For observers on the ground, the sound source comes from the aircraft shell, not from the inside of the aircraft. This principle can be used to explain the principle of invariance of the speed of light, indicating that there is a secondary emission when light waves are switched from one inertial system to another.

Then, the principle of invariance of relative light speed can be expressed as:

$$
\left\{\begin{array}{l}
c_{x}^{\prime}=c_{x} \\
c_{y}^{\prime}=c_{y} \\
c_{z}^{\prime}=c_{z}
\end{array}\right.
$$

Its vector form is:

$$
c^{\prime}=c
$$

It can be seen from the above that the speed of light is constant and does not satisfy the superposition principle. The reason is that when the light wave is switched from one gravitational system (constraint system) to another, there is a secondary emission of the light wave. The speed of light we usually measure is the speed of light measured with the propagation medium of the light wave as the reference system, and the medium of the light wave is always stationary relative to the gravitational field. Therefore, the speed of light measured in any gravitational field is the same. Therefore, we say that the speed of light has nothing to do with the 
selection of the reference frame, and does not mean that the speed of light is the upper limit speed of the universe. We know that the Lorentz transformation is a feasible mathematical expression model for the principle of invariance of the speed of light, and today we have discovered that other mathematical expression models can also explain the principle of invariance of the speed of light. In other words, we have proposed another feasible way of explaining the principle of constant speed of light. (Note: This actually illustrates a problem. The velocity of any wave is measured relative to its propagation medium, and has nothing to do with the selection of the reference system)

Discussion: As can be seen from the above, the propagation medium of light is not absolutely spatially distributed, but is relatively statically distributed relative to different gravitational fields. Therefore, it is consistent with the Michelson-Morley experiment of the medium 'Ether' without absolute spatial distribution.

Of course, for some outstanding scientists, proposing a new explanation for a theory can no longer arouse his interest, and they are more concerned about new predictions. Therefore, in addition to presenting a new explanation on the principle of invariance of the speed of light, this article also predicts that all different types of waves satisfy the principle of invariance of speed. In this way, we can verify the new theory through experiments.

\section{Conclusion}

This paper aims to find a feasible interpretation of the origin of wave-particle duality of microscopic particles, so as to solve the pain points of quantum mechanical probability waves.

In the existing quantum mechanics theory, we believe that microscopic material particles have wave-particle duality. We cannot yet use one of these attributes to fully interpret the other (but we have been trying this kind of work). In this article, I was lucky to find a way to use one of the attributes to interpret the other, and got some new predictions.

\section{Reference}

[1] Gutzwiller M C. Chaos in classical and quantum mechanics[J]. Physics Today, 1991, 44(11):94-96.

[2] Duarte F J. The origin of quantum entanglement experiments based on polarization measurements[J]. The European Physical Journal H, 2012, 37(2):311-318.

[3] Genovese M. Interpretations of Quantum Mechanics and the measurement problem[J]. Journal of Computational \& Theoretical Nanoscience, 2010, 3(3):249.

[4] Mittelstaedt P. The Interpretation of Quantum Mechanics and the Measurement Process[M]// The interpretation of quantum mechanics and the measurement process \% Cambridge University Press, 1998.

[5] Hollowood, Timothy J. The emergent Copenhagen interpretation of quantum mechanics $[\mathrm{J}]$. Journal of Physics A: Mathematical and Theoretical, 2014, 47(18):185301.

[6] Faye J. Copenhagen Interpretation of Quantum Mechanics[J]. Stanford Encyclopedia of Philosophy, 2008.

[7] Dewitt B S, Graham N. The Many Worlds Interpretation of Quantum Mechanics[M]. Princeton University Press, 1973.

[8] Adler S L. Why Decoherence has not Solved the Measurement Problem: A Response to P. W. Anderson[J]. Studies in History \& Philosophy of Modern Physics, 2001, 34(1):135-142.

[9] Tammaro E. Why Current Interpretations of Quantum Mechanics are Deficient[J]. Enprint Arrive, 2014.

[10] Rowlands P. Quantum Uncertainty, Wave-Particle Duality and Fundamental Symmetries[M]// The Present Status of the Quantum Theory of Light. Springer Netherlands, 1997.

[11] Roy S. The Present Status of the Quantum Theory of Light[M]// The present status of the quantum theory of light: Kluwer Academic Pub. 1997.

[12] Bassi E B A, Dorr, Detlef, Weber T, et al. Quantum Mechanics: Are there Quantum Jumps? and On the Present Status of Quantum Mechanics[J]. 2006.

[13] Rashkovskiy S A. Is a rational explanation of wave-particle duality possible? [J]. 2013

[14] Jarosław Pykacz. Attempt at the Logical Explanation of the Wave-Particle Duality[J]. 1999.

[15] Lai M, Diels J C. Wave-particle duality of a photon in emission[J]. Journal of the Optical 
Society of America B, 1992, 9(12):2290-2294.

[16] Gatti A, Brambilla E, Lugiato L A. Entangled imaging and wave-particle duality: from the microscopic to the macroscopic realm[C]// European Quantum Electronics Conference. IEEE, 2003.

[17] Mandel L, Wolf E, Shapiro J H. Optical Coherence and Quantum Optics[J]. Physics Today, 1996, 49(5):68-70. 